\title{
Comparison of unprocessed silk cocoon and silk cocoon middle layer membranes for guided bone regeneration
}

\author{
Seong-Gon Kim", Min-Keun Kim', HaeYong Kweon², You-Young Jo², Kwang-Gill Lee ${ }^{2}$ and Jeong Keun Lee ${ }^{3}$
}

\begin{abstract}
Background: Silk cocoon is composed of multiple layers. The natural silk cocoon containing all layers was cut as a rectangular shape as defined as total group. The inner and outermost layers were removed from the total group and the remained mat was defined as the middle group. The objectives of this study was to compare the total group with the middle group as a barrier membrane for the guided bone regeneration.

Methods: The effects of these materials on the cellular proliferation and alkaline phosphatase (ALP) expression of MG63 cells were explored. For comparing bone regeneration ability, bilateral bone defects were created in calvarial areas in ten adult New Zealand white rabbits. The defects were covered with silk membranes of the middle group, with silk membrane of the total group used as the control on the contralateral side. The defects were allowed to heal for 4 and 8 weeks. Micro-computerized tomography $(\mu \mathrm{CT})$ and histological examination were performed.
\end{abstract}

Results: The middle group exhibited a higher MTT value 48 and $72 \mathrm{~h}$ after treatment compared to the total group. ALP expression was also higher in the middle group. The results of $\mu \mathrm{CT}$ and histologic examination showed that new bone formation was significantly higher in the middle group compared to the total group 8 weeks postoperatively $(P<0.05)$.

Conclusions: In conclusion, the middle layer of the silk cocoon supports guided bone regeneration better than unprocessed silk cocoon.

Keywords: Guided bone regeneration, Silk cocoon, Membrane, Bone defect

\section{Background}

Sufficient alveolar bone volume is a prerequisite for dental implant treatment for edentulous patients, assisted by direct bone regeneration. Guided bone regeneration (GBR) is a technique that compensates for the lost alveolar bone, in which a membrane acts as a barrier to block epithelial migration [1]. The idea of a barrier membrane dates back to Bassett et al., who designed cellulose acetate filters to support the regeneration of nerves and tendons [2]. The concept of guided bone regeneration originated with attempts to gain space for the regeneration of osseous defects via the utilization of the barrier membrane [3]. Ideal GBR membranes require

\footnotetext{
* Correspondence: epker@chol.com

${ }^{1}$ Department of Oral and Maxillofacial Surgery, College of Dentistry,

Gangneung-Wonju National University, Gangneung, Gangwondo 210-702,

Republic of Korea

Full list of author information is available at the end of the article
}

adequate mechanical stability in addition to optimal biological properties [4].

A variety of membranes are commercially available for use in the clinic, including bioresorbable and nonresorbable membranes, with collagen membranes and titanium-reinforced polytetrafluoroethylene (Gore-Tex ${ }^{\circ}$ ) as typical examples of these two types, respectively. Bioresorbable collagen membranes, mainly prepared from porcine collagen [5], are mechanically weak and are unable to maintain space [6], whereas the non-resorbable Gore-Tex ${ }^{\circ}$ has sufficient mechanical strength to support space even for vertical bone augmentation [7]. Despite this advantage of providing support to the osseous defect, the effectiveness of Gore-Tex ${ }^{\circ}$ is limited due to the risk of membrane exposure leading to bone graft failure [4]. For the manufacturing collagen membranes, cross-linking agents, such as diphenylphosphorylazide or glutaraldehyde, have been used $[8,9]$. Cross-linking 
agents can be cytotoxic during the healing phase [10]. With respect to the mechanical properties, Gore-Tex ${ }^{\circ}$ membrane is easily torn in wet conditions and difficult to adapt and suture [11].

The aim of this study was to overcome the shortcomings of the existing membranes by developing new barrier membrane from silk protein. Silk is a natural macromolecule originating from Bombyx mori that mainly consists of two proteins, fibroin and sericin [12-15]. Fibroin is the structural center of silk, with a specific fibroin layer giving the silk tensile strength. The high glycine and alanine content in silk fibroin leads to tightly packed beta membranes, resulting in rigidity and tensile strength [15]. Silk fibroin can increase alkaline phosphatase activity and type I collagen expression in MG63 cells [16]. As both proteins are bone formation marker, silk fibroin has been widely used as a scaffold for bone graft [12]. The first silk barrier membrane was produced by electrospinning, which requires a large facility for mass production [17]. However, silk fibroin film was found to be fragile and dissolved easily in aqueous solution when it was used a barrier membrane [18].

Sericin is the gum coating the surface of fibroin, allowing fibroin molecules to stick to each other. Sericin is under investigation as a wound dressing material [19], and silk sericin-capped silver nanoparticles exhibited antibacterial activity against many gram-positive bacteria and gramnegative bacteria. Recent reports on the non-toxic nature of sericin [20] supported the use of whole silkworm-cocoonderived silk membranes [21]. Because of an early report that sericin evoked IgE and IgG antibody reactions in humans [22], silk fibroin was purified from sericin by boiling silk cocoons in an alkaline solution (degumming) to remove the source of the immunologic reaction. In this study, we deliberately omitted the degumming process to avoid removing sericin. Silk from the cocoons of silkworms are known to have extraordinary mechanical properties [23], and the mechanical peeling of the silk cocoon generates membranes of different thicknesses [24], each of which could be utilized as barrier membranes. Our previous studies compared silkworm-cocoon-derived silk membranes with existing barrier membranes [25] and studied the effect of thickness [21]. Silkworm-cocoon-derived silk membranes showed comparable ability of bone regeneration compared to collagen and Gore-Tex membrane [25]. Thicker silkworm-cocoon-derived silk membranes had better bone formation than the thinner one [21]. However, these studies used only the middle portion of the silkworm cocoon. Therefore, the process for removing the inner and the outermost layers of the silkworm cocoon is still needed. However, the reason of removing the inner and the outermost portions is unclear. At first, they were removed because of contamination from the moth pupas or the outer environment. This may be cleaned by several methods without removing the layers. If the natural cocoon has similar bone formation ability to that of the middle portion, removing the inner and the outermost layers of silkworm cocoon will not be needed. This will simplify the production process and reduce the cost for the production.

Silk cocoon is composed of multiple layers. After removing the inner and outermost layers, the remained membrane was assigned as the middle group. The objective of this study was to compare natural silk cocoons with the membranes of the middle group as barrier membranes for guided bone regeneration. The membranes were examined by scanning electron microscopy and osteoblast-like cell culture. The silk membranes were grafted into the calvarial bone defects of rabbits, and the new bone formation of each group was compared by micro-computerized tomography $(\mu \mathrm{CT})$ and histological examination.

\section{Methods \\ Silk membrane}

B. mori cocoons were obtained from the Rural Development Administration (Jeonju, Korea). The raw cocoons were cut to $1 \mathrm{~cm} \times 1-\mathrm{cm}$ size pieces and used for the total group. The inner and outermost layers of the cocoons were removed by mechanical peeling (Fig. 1). The thickness of the removed portion was approximately $0.2 \mathrm{~mm}$ for each. Then, the remaining middle layer was cut as $1 \mathrm{~cm} \times 1 \mathrm{~cm}$ and used for the middle group. Each membrane was then washed with ethanol. Subsequently, the silk membranes were sterilized by autoclave at $121{ }^{\circ} \mathrm{C}$ and 1.5 atmospheric pressure for $30 \mathrm{~min}$. The thickness was measured by caliper.

\section{SEM imaging}

All materials were prepared for scanning electron microscope (SEM) examination. The prepared specimens underwent SEM analysis at Gangneung Center in Korea Basic Science Institute (KBSI). After immobilization of the samples on the plate, each sample was coated with gold. Specimens were imaged using a SU-70 microscope (Hitachi, Japan) operating at $5 \mathrm{keV}$.

\section{Cell culture}

In vitro tests were performed using MG-63 osteoblast-like cells (ATCC, Manassas, VA, USA). The cells were grown to $80 \%$ confluence in Dulbecco's modified Eagle's medium-high glucose (PAA Laboratories, Linz, Austria) containing $1 \%$ penicillin/streptomycin (100x), supplemented with $10 \%$ fetal bovine serum (PAA Laboratories, Etobicoke, ON, Canada) at $37{ }^{\circ} \mathrm{C}$ in an atmosphere of $5 \%$ $\mathrm{CO}_{2}$ and $99 \%$ relative humidity. Media were changed every 3 days.

The mat from the total group and middle group was cut as $1 \mathrm{~cm}^{2}$-sized rectangular shape. The samples were 


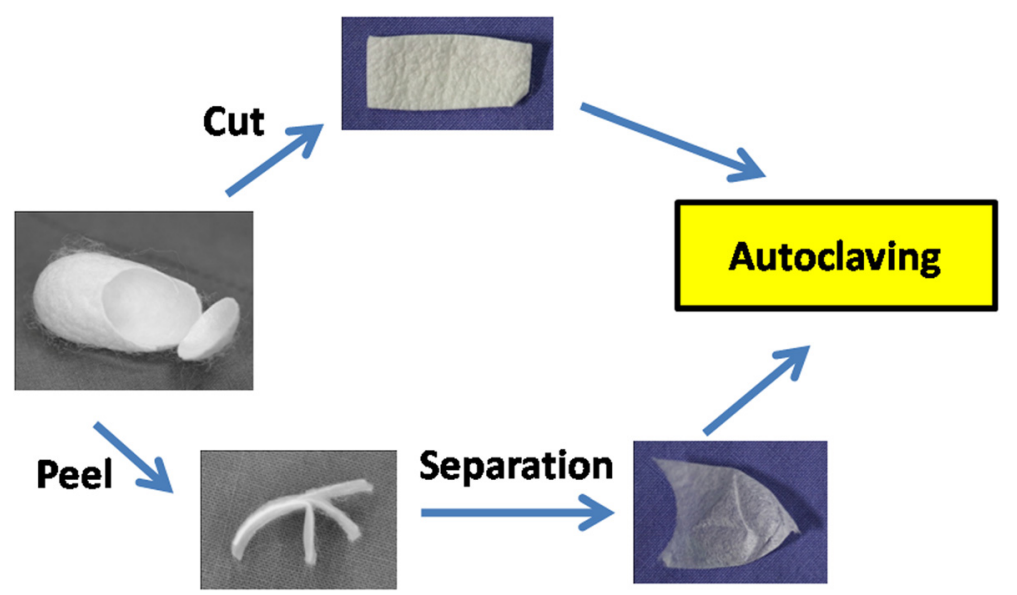

Fig. 1 Schematic drawings for the silk membrane fabrication

sterilized by autoclave at $121{ }^{\circ} \mathrm{C}$ and 1.5 atmospheric pressure for $30 \mathrm{~min}$ and passivated in DMEM culture medium for $12 \mathrm{~h}$, prior to seeding cells. A seeding density of 20,000 cells $/ \mathrm{cm}^{2}$ was used, and the specified amounts of cells were seeded using $1 \mathrm{ml}$ of DMEM medium. The plates were incubated in a humidified environment for a period of up to 14 days, and the medium was changed every second day. At specific time intervals, samples were analyzed for tetrazolium salt 3-(4, 5-dimethylthiazole-2-yl)-2,5-diphenyltetrazolium bromide (MTT) assay and alkaline phosphatase (ALP) assay.

\section{MTT assay and alkaline phosphatase assay}

Cell viability was quantified after 24,48 , and 96 h of culture growth using the tetrazolium salt MTT assay. Briefly, cells were incubated with MTT solution (Cell proliferation kit I; Roche Molecular Biochemicals, Mannheim, Germany) in six-well plates for $4 \mathrm{~h}$ at $37{ }^{\circ} \mathrm{C}$ in an atmosphere of $5 \%$ $\mathrm{CO}_{2}$ and $99 \%$ relative humidity. Formazan crystals were solubilized with DMSO at room temperature overnight, and the product concentration was determined by measuring the absorbance at $540 \mathrm{~nm}$ with a Victor Multilabel counter (Perkin-Elmer-Wallac, Freiburg, Germany).

ALP was evaluated by the transformation of $\mathrm{p}$ nitrophenylphosphate into p-nitrophenol at $37{ }^{\circ} \mathrm{C}$ and $\mathrm{pH} 10.2$ using appropriate reactive chemicals (all from Sigma, St. Louis, MO, USA), while the specific activity of ALP was calculated based on the protein concentration of lysates determined using a commercially available colorimetric assay (\#71230, AnaSpec, Freemont, CA, USA). The product concentration was determined by measuring the absorbance at $415 \mathrm{~nm}$ with an iMark microplate absorbance reader (BioRad, Hercules, CA, USA). ALP standard was prepared in a serial dilution of 1:50 and detected using an absorbance of $405 \mathrm{~nm}$.

\section{Animals and surgical procedure}

Ten 12-week-old New Zealand white rabbits with an average weight of $2.3 \mathrm{~kg}$ (range $2.0-2.5 \mathrm{~kg}$ ) were used for this experiment. This experiment was approved by the Institutional Animal Care and Use Committee of the Gangneung-Wonju National University, Gangneung, Korea (GWNU-2014-5).

General anesthesia was administered by intramuscular injection of a combination of Zoletil $50(15 \mathrm{mg} / \mathrm{kg}$; Vibac, Carros, France) and Rumpun $(0.2 \mathrm{~mL} / \mathrm{kg}$; Bayer Korea, Seoul, Korea). The cranial area was shaved and disinfected with povidone-iodine. Two percent lidocaine with epinephrine $(1: 100,000)$ was applied to the cranial area. A midline incision was made in the sagittal plane of the skull, and subperiosteal dissection was performed to expose the calvaria. A trephine bur was used under saline irrigation to create a calvarial defect. Two 8-mmdiameter defects were created, one on each side of the midline. The calvarial defects were covered with the total or middle membranes.

Following treatment, the pericranium and skin were closed in layers with 3-0 black silk (AILEE, Busan, Korea). After surgery, the rabbits received $1 \mathrm{mg} / \mathrm{kg}$ gentamicin (Kookje, Seoul, Korea) and $0.5 \mathrm{~mL} / \mathrm{kg}$ pyrin (Green Cross Veterinary Products, Seoul, Korea) intramuscularly, three times daily for 3 days.

Each rabbit was individually caged and received food and water. All animals were killed at 4 or 8 weeks after surgery. Specimens were separated and fixed in $10 \%$ formalin. After $\mu \mathrm{CT}$ analysis, histological analysis was performed.

\section{$\mu \mathrm{CT}$ analysis}

The prepared specimens were analyzed by $\mu \mathrm{CT}$ using an animal PET/CT/SPECT system (Inveon, Siemens, Erlangen, Germany) at the Ochang Center of the Korea Basic Science Institute. The $\mu \mathrm{CT}$ scanner was set to $80 \mathrm{kV}$ for the X-ray tube, with a $500-\mu \mathrm{A}$ current for the X-ray source and a 
210-ms exposure time. The detector and X-ray source were rotated through $360^{\circ}$ in 360 steps, with 30 calibration exposures. The system magnification was set to produce an axial field of view (FOV) of $30.74 \mathrm{~mm}$ and a trans-axial FOV of $30.74 \mathrm{~mm}$. The scanned images were reconstructed using the Inveon Research Workplace software (Siemens). Gross profiles of the specimens were obtained from reconstructed three-dimensional images. Because the initial defect was round in shape with an $8.0-\mathrm{mm}$ diameter, the region of interest (ROI) was set based on the initial defect size and shape. The ROI of each specimen was analyzed for bone volume (BV).

\section{Histomorphometric evaluation}

The calvarial samples were harvested, decalcified in $5 \%$ nitric acid for 5 days, and dehydrated in ethyl alcohol and xylene. After separation of the parietal bones through the midline sagittal suture, the calvarial samples were embedded in paraffin blocks. The paraffin blocks were sliced into sections that were then stained with hematoxylin and eosin. The section with the largest defect area was selected, along with sections $50 \mu \mathrm{m}$ proximal and distal to the largest defect section.

The staining procedure for hematoxylin and eosin staining was as follows. First, de-wax and hydrate paraffin sections. The slide was stained in hematoxylin for $5 \mathrm{~min}$. Overstained sections can easily be differentiated by agitating for a second in acid-alcohol, then washing in tap water for $5 \mathrm{~min}$. The slides were immersed in eosin for $30 \mathrm{~s}$ and then wash them in running tap water for $1 \mathrm{~min}$. The slides were dehydrated and clear in xylene.

Digital images of the selected sections were captured with a digital camera (DP-73; Olympus, Tokyo, Japan). The images were analyzed by Sigma Scan pro (SPSS, Chicago, IL). The new bone formation was calculated as the percentage of newly formed bone in the calvarial defect area.

\section{Statistical analysis}

SPSS for window ver. 19 (IBM Co., Armonk, NY, USA) were used for statistical analysis. The differences between the mean values of the total group and the middle group in the MTT and alkaline phosphatase (ALP) assays were evaluated by independent sample $t$ tests. Paired $t$ test was used for comparison the samples within the same animal. The level of significance was set as $P<0.05$.

\section{Results}

\section{Gross appearance}

The thickness of the total group was $0.61 \pm 0.02 \mathrm{~mm}$ and that of the middle group was $0.32 \pm 0.03 \mathrm{~mm}$. Gross morphology was observed by SEM. No striking differences in morphology were observed between groups (Fig. 2).
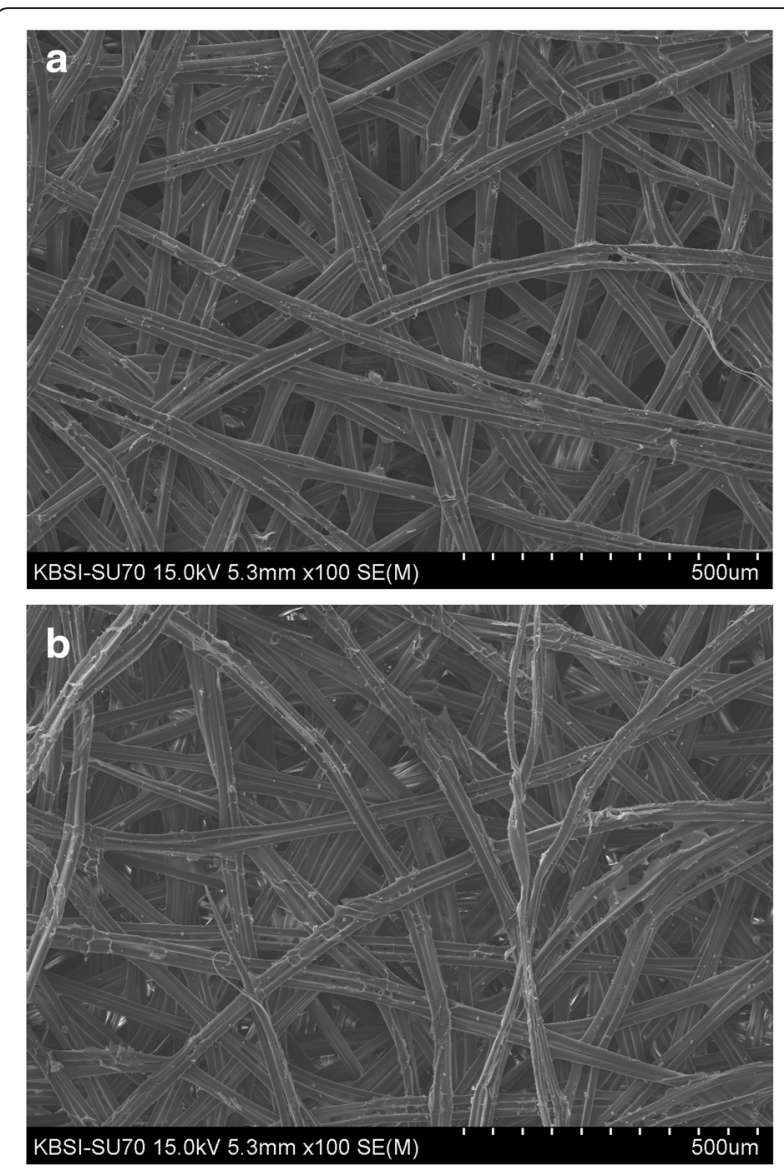

Fig. 2 SEM images of the silk membrane. a Unprocessed control. b Middle layer group

\section{MTT assay and ALP assay}

Compared with the total group, MG-63 cells treated with the middle layer exhibited decreases in MTT activity at $24 \mathrm{~h}$ after application $(P<0.001)$. MG-63 cells treated with the middle layer exhibited increases in MTT activity at 48 and $96 \mathrm{~h}$ after appliscation $(P<0.001)$ (Fig. 3a).

The relative ALP activity of the middle group was approximately threefold higher compared with that of the total group. The difference between groups was statistically significant $(P<0.001)$ (Fig. $3 b)$.

\section{In vivo test}

The results of the $\mu \mathrm{CT}$ analysis are presented in Fig. $4 \mathrm{a}$. The average values of the two measured variables were higher in the middle membrane group than those in the total membrane group at both 4 and 8 weeks. The bone volume in the middle group at 4 weeks after the operation was $5.85 \pm 6.94 \mathrm{~mm}^{3}$, and the volume in the total group was $2.20 \pm 2.81 \mathrm{~mm}^{3}$. This difference was not statistically significant $(P>0.05)$. The bone volume in the middle group at 8 weeks after the operation was $11.17 \pm 5.69 \mathrm{~mm}^{3}$ and 

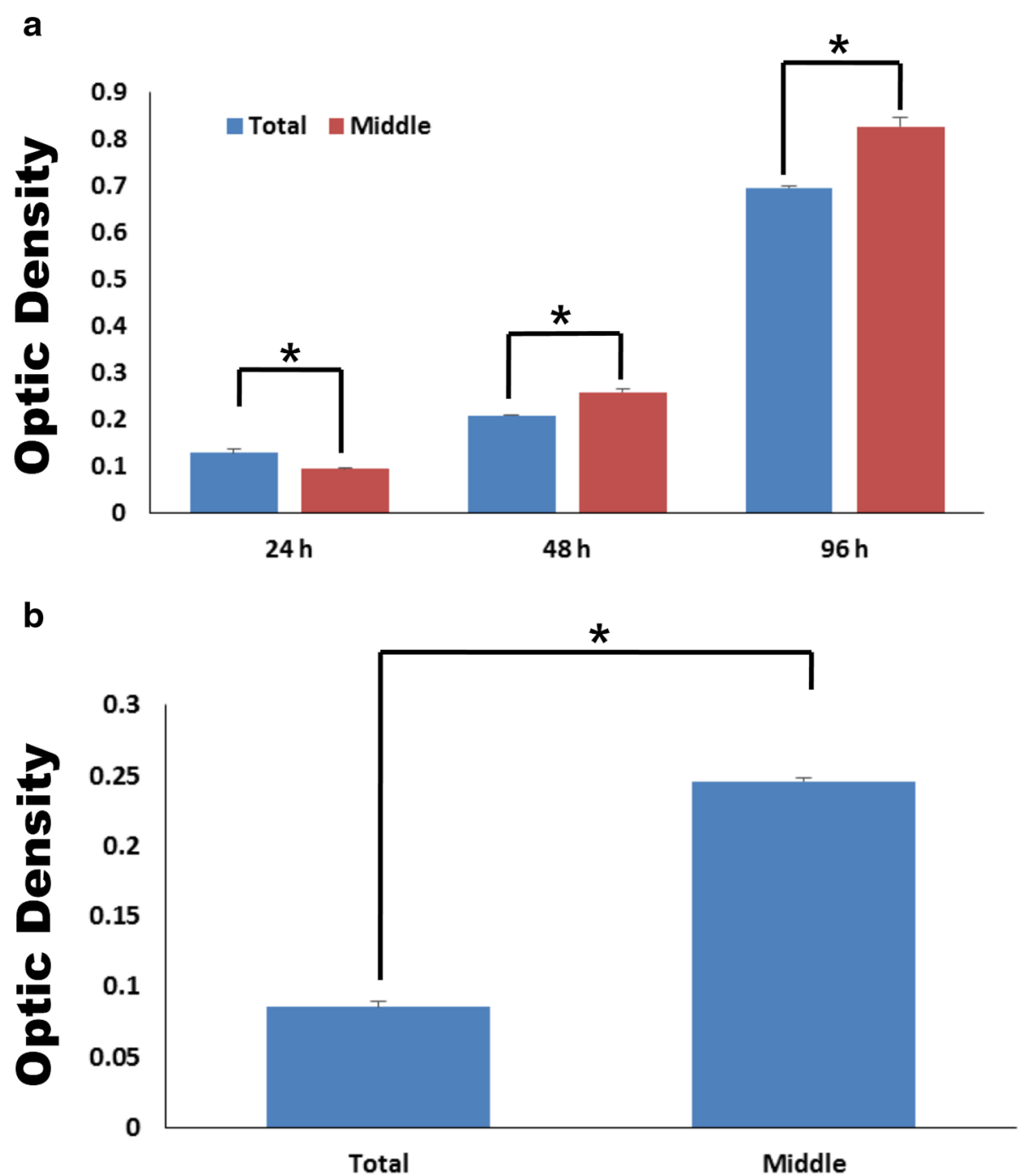

Fig. 3 The biological evaluations. a MTT assay of MG63s on unprocessed silk cocoon (total group) and middle layer silk cocoon (middle group) membranes (after 24, 48, 72 h of cell culture, asterisk significantly different at $P<0.05$ ) and $\mathbf{b}$ alkaline phosphatase assay of MG63s after $72 \mathrm{~h}$ of cell culture (asterisk significantly different at $P<0.05$ ). Error bars mean standard deviation

that in the total group was $4.73 \pm 5.38 \mathrm{~mm}^{3}$. This difference was statistically significant $(P=0.048)$ (Fig. 4b).

The results of the histological analysis are presented in Fig. 5. The average values of the two measured variables were higher in the middle membrane group than those in the total membrane group at both 4 and 8 weeks. The new bone formation in the middle group at 4 weeks after the operation was $13.14 \pm 13.23 \%$ and that in the total group was $3.32 \pm 2.23 \%$. This difference was not statistically significant $(P>0.05)$. The new bone formation in the middle group at 8 weeks after the operation was $22.41 \pm 8.38 \%$ and that in the total group was $8.94 \pm 5.16 \%$. This difference was statistically significant $(P=0.016)$.

Less residual membrane was observed in the specimen at 8 weeks after the operation compared to the specimen at
4 weeks after the operation in both groups (Fig. 6a, b). No foreign body giant cells were observed in either group (Fig. 6e, f). Interestingly, new bone formation was observed above the membrane in the middle group 8 weeks after the operation (Fig. 6c, g). In the total group, new bone formation was observed on both sides of the membrane (Fig. 6d, h).

\section{Discussion}

This study found that the middle layer of silk cocoon induced better new bone formation than unprocessed silk cocoon. As the process of separating the middle layer did not involve any chemical treatment, the gross morphology observed by SEM was similar between the two groups (Fig. 2). As the membrane from the middle layer of the cocoon was thinner than the unprocessed 


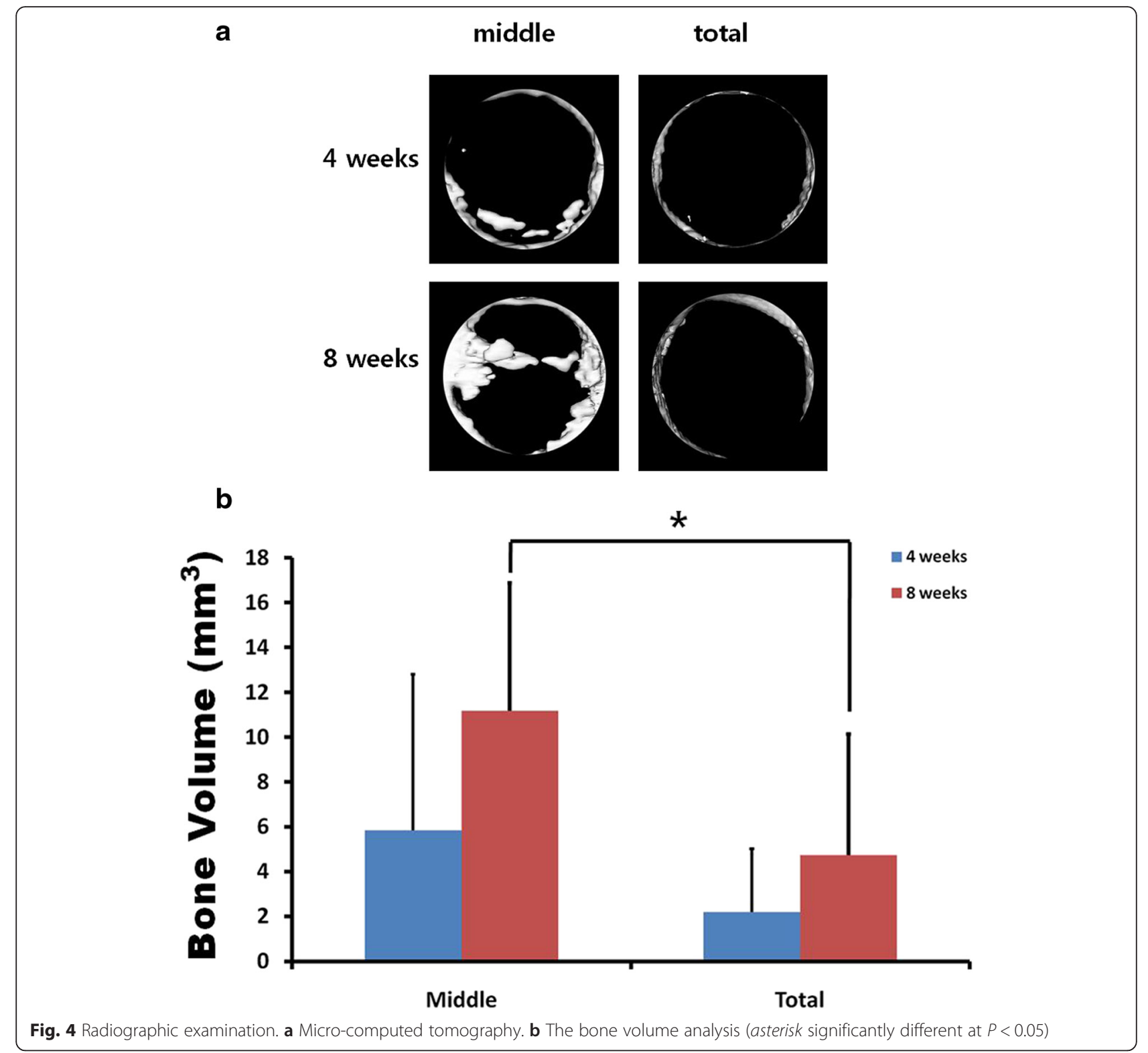

silk cocoon, dentists may find it easier to handle the middle group membrane. To the best of our knowledge, no reports have compared the unprocessed silk cocoon and the middle layer of silk cocoon in the GBR technique.

MG-63 cells treated with the middle layer of the cocoon of B. mori showed decreased MTT activity $24 \mathrm{~h}$ after treatment, but the activities were increased at 48 and $96 \mathrm{~h}$ (Fig. 3a, $P<0.001$ ). These results for the middle group were statistically significant compared to those of the total group, which shows that the overall cellular toxicity of the middle group is favorable. The relative ALP activity of the middle group was dramatically higher than that of the total group, and this result was statistically significant (Fig. 3b, $P<0.001$ ). Taking these results of the cellular studies into consideration, the silk membrane made of the middle layer of the cocoon of $B$. mori exhibits superior safety and efficacy to the membrane prepared from total cocoon.

The results of the animal study were similar to those of the cellular experiments. The results of the $\mu \mathrm{CT}$ analysis showed that the bone volume in the middle group was significantly higher than that in the total group at 8 weeks postoperatively (Fig. $4, P=0.048$ ). The histological analysis demonstrated significantly greater new bone formation at 8 weeks after the operation in the middle group compared with the total group (Fig. 5, $P=0.016)$. The inner layer of silk cocoon has a lower porosity and a smaller average diameter of silk than the outer layer [24]. Each layer of the silk cocoon has a different silk fibroin to sericin ratio [26]. The outermost 


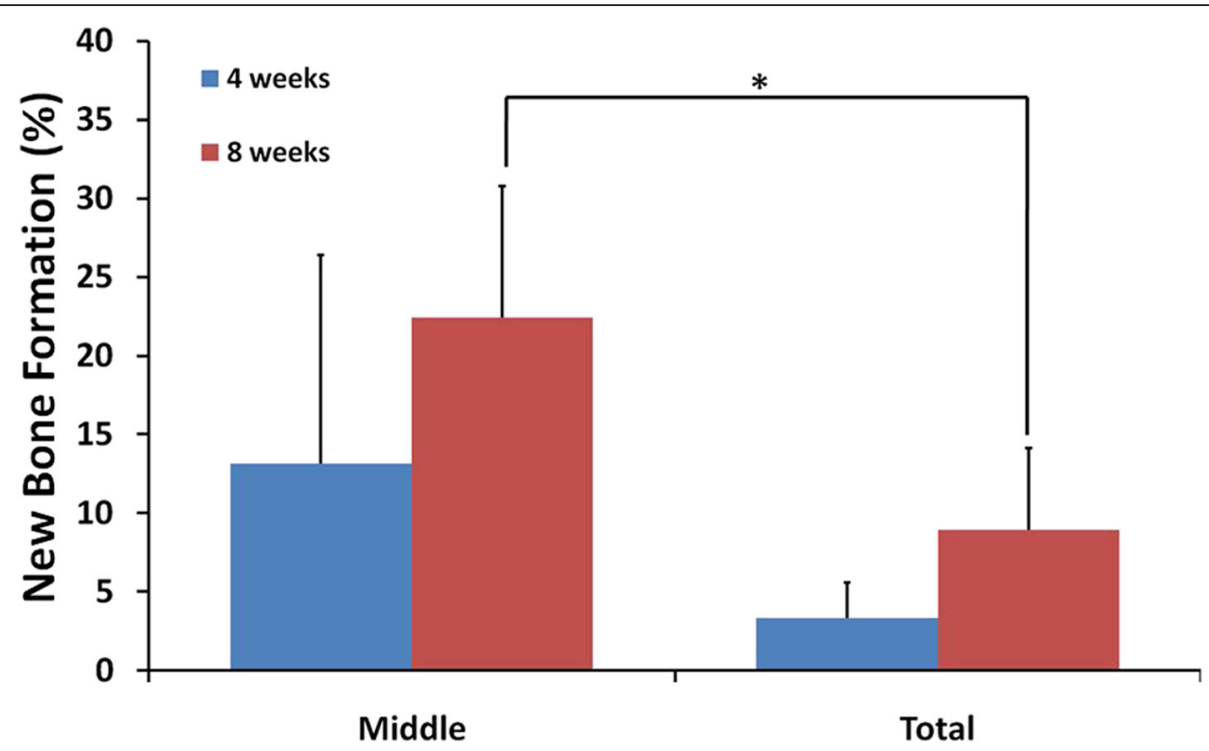

Fig. 5 Histological analysis of new bone formation (asterisk significantly different at $P<0.05, n=5$ )

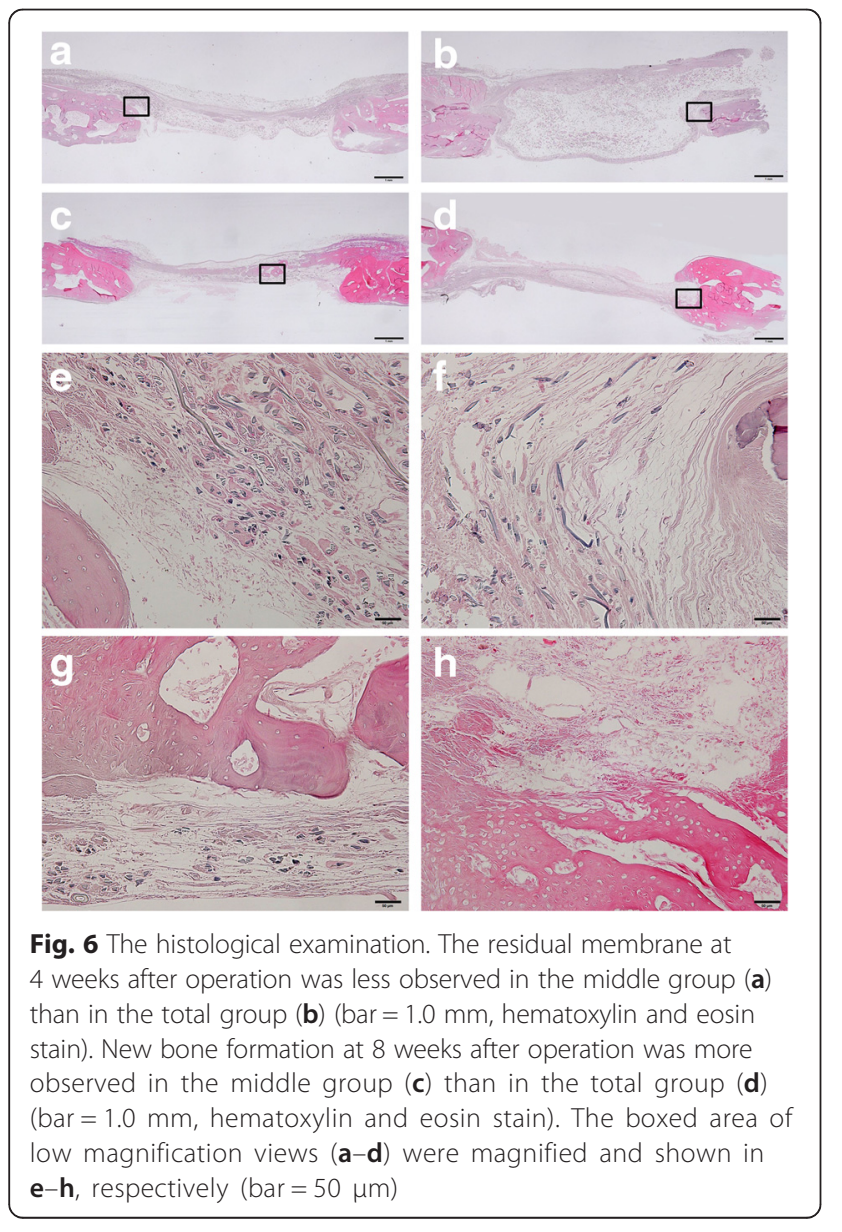

layer has over $30 \%$ of sericin, and the inner most layer has less than $20 \%$ of sericin [26]. Besides silk fibroin and sericin, other natural impurities, such as waxes for protecting the moth pupas are also found [26]. The antiinflammatory effects of sericin [27] may also induce immune reactions upon physical association with fibroin-silk fibers [28]. The graft composition determines whether sericin exerted a positive or negative effect on graft success. The middle layer induced the best new bone formation among divided silk cocoon layers (Fig. 6g). The middle layer may have the optimal composition ratio of silk fibroin and sericin for bone formation. Comparing between different thicknesses of the same silk cocoon middle layer, the thicker membrane leads to better new bone formation [21]. Therefore, the silk mat from the middle layer only seemed to be proper for GBR technique. The inner and outermost layers of the silk cocoon should be removed before use for the GBR technique. Except for the different amino acid composition among layers, other differences among layers are still unclear. The exact mechanism should be clarified by further studies.

Less residual membrane was observed in the specimen 8 weeks after operation compared to the specimen 4 weeks after operation in both groups (Fig. 6). As the membranes derived from silk cocoon are composed of amino acid, they may underwent proteolysis gradually. Other membranes based on natural materials, such as collagen, are also biodegradable [17]. In case of collagen membrane, if it is not exposed to the oral environment, it is intact in the graft site until 28 days after implantation [29]. No foreign body giant cells were observed in either group. Interestingly, most previous silk fibroin grafts have elicited many foreign body giant cells. Sericin 
exerts an anti-inflammatory effect [27]. Sericin or other components of the silk cocoon may inhibit foreign body giant cell formation. In this study, both membranes had been sterilized by autoclave, and their mechanical properties after autoclaving were proved to be intact by the measured stress-strain curve, indicating that the membranes were not damaged by autoclaving (data not shown). Therefore, the silk cocoon-derived membrane can be used for clinical trials after sterilization.

\section{Conclusions}

In conclusion, the middle layer of the silk cocoon was more appropriate for use in the GBR technique than the unprocessed silk cocoon. However, few data are available on the clinical applications of silk membranes. As the processing procedure used here is simple and natural, clinical trials should be carried out using materials prepared using this procedure.

\section{Abbreviations \\ ALP: alkaline phosphatase; GBR: guided bone regeneration; MTT: tetrazolium salt 3-(4, 5-dimethylthiazole-2-yl)-2,5-diphenyltetrazolium bromide; SEM: scanning electron microscope; $\mu C \mathrm{C}$ : micro-computerized tomography.}

\section{Competing interests}

The authors declare that they have no competing interests.

\section{Authors' contributions}

KMK did most of the experiment, and KHY designed this experiment. JYY and LKG developed and set up the materials. KSG and LJK wrote the manuscript and did critical review on the experimental process. All authors read and approved the final manuscript.

\section{Acknowledgements}

This work was supported by a grant from the Next-Generation BioGreen21 Program (Center for Nutraceutical and Pharmaceutical Materials no. PJ01121404), Rural Development Administration, Republic of Korea.

\author{
Author details \\ 'Department of Oral and Maxillofacial Surgery, College of Dentistry, \\ Gangneung-Wonju National University, Gangneung, Gangwondo 210-702, \\ Republic of Korea. ${ }^{2}$ Sericultural and Apicultural Materials Division, National \\ Academy of Agricultural Science, Suwon, South Korea. ${ }^{3}$ Department of Oral \\ and Maxillofacial Surgery, Ajou University School of Medicine, Suwon, South \\ Korea.
}

Received: 8 January 2016 Accepted: 17 February 2016

Published online: 29 February 2016

\section{References}

1. Hämmerle $\mathrm{CH}$, Jung RE, Feloutzis A (2002) A systematic review of the survival of implants in bone sites augmented with barrier membranes (guided bone regeneration) in partially edentulous patients. J Clin Periodontol 29:226-231

2. $\quad$ Bassett CA, Campbell JB, Girado JM, Rossi JP, Seymour RJ (1956) Application of monomolecular filter tubes in bridging gaps in peripheral nerves and for prevention of neuroma formation; a preliminary report. J Neurosurg 13:635-637

3. Dahlin C, Linde A, Gottlow J, Nyman S (1988) Healing of bone defects by guided tissue regeneration. Plast Reconstr Surg 81:672-676

4. Dimitriou R, Mataliotakis Gl, Calori GM, Giannoudis PV (2012) The role of barrier membranes for guided bone regeneration and restoration of large bone defects: current experimental and clinical evidence. BMC Med 10:81

5. Rothamel D, Schwarz F, Sculean A, Herten M, Scherbaum W, Becker J (2004) Biocompatibility of various collagen membranes in cultures of human PDL fibroblasts and human osteoblast-like cells. Clin Oral Implants Res 15:443-449
6. Lundgren D, Sennerby L, Falk H, Friberg B, Nyman S (1994) The use of a new bioresorbable barrier for guided bone regeneration in connection with implant installation. Case reports. Clin Oral Implants Res 5:177-184

7. Roccuzzo M, Ramieri G, Spada MC, Bianchi SD, Berrone S (2004) Vertical alveolar ridge augmentation by means of a titanium mesh and autogenous bone grafts. Clin Oral Implants Res 15:73-81

8. Zahedi S, Legrand R, Brunel G, Albert A, Dewé W, Coumans B et al (1998) Evaluation of a diphenylphosphorylazide-crosslinked collagen membrane for guided bone regeneration in mandibular defects in rats. J Periodontol 69:1238-1246

9. Rothamel D, Schwarz F, Sager M, Herten M, Sculean A, Becker J (2005) Biodegradation of differently cross-linked collagen membranes: an experimental study in the rat. Clin Oral Impl Res 16:369-378

10. Speer DP, Chvapil M, Eskelson CD, Ulreich J (1980) Biological effects of residual glutaraldehyde in glutaraldehyde-tanned collagen biomaterials. J Biomed Mater Res 14:753-764

11. Zhao S, Pinholt EM, Madsen JE, Donath K (2000) Histological evaluation of different biodegradable and non-biodegradable membranes implanted subcutaneously in rats. J Craniomaxillofac Surg 28:116-122

12. Li C, Vepari C, Jin HJ, Kaplan DL (2006) Electrospun silk-BMP-2 scaffolds for bone tissue engineering. Biomaterials 27:3115-3124

13. Kim MK, Yoo KY, Kwon KJ et al (2014) Powdered wound dressing materials made from wild silkworm Antheraea pernyi silk fibroin on full-skin thickness burn wounds on rats. Maxillofac Plast Reconstr Surg 36:111-115

14. Park YT, Kweon HY, Kim SG (2014) Soft tissue augmentation with silk composite graft. Maxillofac Plast Reconstr Surg 36:192-200

15. Valluzzi R, Gido SP, Muller W, Kaplan DL (1999) Orientation of silk III at the air-water interface. Int J Biol Macromol 24:237-242

16. Kim JY, Choi JY, Jeong JH, Jang ES, Kim AS, Kim SG et al (2010) Low molecular weight silk fibroin increases alkaline phosphatase and type I collagen expression in MG63 cells. BMB Rep 43:52-56

17. Lee SW, Kim SG (2014) Membranes for the guided bone regeneration. Maxillofac Plast Reconstr Surg 36:239-246

18. Song JY, Kim SG, Lee JW, Chae WS, Kweon H, Jo YY et al (2011) Accelerated healing with the use of a silk fibroin membrane for the guided bone regeneration technique. Oral Surg Oral Med Oral Pathol Oral Radiol Endodontol 112:e26-e33

19. Retzepi M, Donos N (2010) Guided bone regeneration: biological principle and therapeutic applications. Clin Oral Implants Res 21:567-576

20. Aramwit P, Kanokpanont S, De-Eknamkul W, Srichana T (2009) Monitoring of inflammatory mediators induced by silk sericin. J Biosci Bioengineer 107: 556-561

21. Seok H, Kim MK, Kim SG, Kweon H (2014) Comparison of silkworm-cocoonderived silk membranes of two different thicknesses for guided bone regeneration. J Craniofac Surg 25:2066-2069

22. Dewair M, Baur X, Ziegler K (1985) Use of immunoblot technique for detection of human lgE and IgG antibodies to individual silk proteins. J Allergy Clin Immunol 76:537-542

23. Vepari C, Kaplan DL (2007) Silk as a biomaterial. Progr Polym Sci 32:991-1007

24. Zhao HP, Feng XQ, Yu SW, Cui WZ, Zou FZ (2005) Mechanical properties of silkworm cocoons. Polymer 46:9192-9201

25. Ha YY, Park YW, Kweon HY, Jo YY, Kim SG (2014) Comparison of the physical properties and in vivo bioactivities of silkworm-cocoon-derived silk membrane, collagen membrane, and polytetrafluoroethylene membrane for guided bone regeneration. Macromol Res 22:1018-1023

26. Mondal M, Trivedy K, Nirmal Kumar S (2007) The silk proteins, sericin and fibroin in silkworm, Bombyx mori Linn._-a review. Caspian J Env Sci 5:63-76

27. Aramwit $P$, Towiwat $P$, Srichana $T$ (2013) Anti-inflammatory potential of silk sericin. Nat Prod Commun 8:501-504

28. Panilaitis B, Altman GH, Chen J, Jin HJ, Karageorgiou V, Kaplan DL (2003) Macrophage responses to silk. Biomaterials 24:3079-3085

29. Tal H, Kozlovsky A, Artzi Z, Nemcovsky CE, Moses O (2008) Cross-linked and non-cross-linked collagen barrier membranes disintegrate following surgical exposure to the oral environment: a histological study in the cat. Clin Oral Implants Res 19:760-766 